Research Article

\title{
Finding Minimum Distance on Birkhoff-James in Banach Space
}

\author{
Susilo Hariyanto ${ }^{1, *}$, Titi Udjiani ${ }^{1}$, Yuri C Sagala ${ }^{1}$, Muhammad Rafid Fadil ${ }^{1}$ \\ 1 Department of Mathematics, Diponegoro University \\ * Corresponding author: sus2_hariyanto@yahoo.co.id
}

Received: 20 June 2020; Accepted: 15 July 2020; Published: 20 July 2020

\begin{abstract}
In this paper we define orthogonality concept on Banach space. That is called Birkhoff-James orthogonality. Some new problem about the correlation of orthogonality between Hilbert space and Birkhoff-James were discussed. Correlation investigated by using particular norm. In other side, correlation of minimum distance in Banach space and Birkhoff-James orthogonality also discussed, by generalizing minimum distance in Hilbert space.
\end{abstract}

Keywords: Banach space, Birkhoff-James orthogonality, minimum distance

\section{Introduction}

Functional analysis is one of the abstract branches in mathematics which studies about space structure (such as vector space, Banach space, Hilbert space, and so on) and its mapping between these spaces (a basic concept that important in this functional analysis). In this study, we also involve topology so it is related to the concept of continuity and convergence of sequences in this space. This is what distinguishes between functional analysis and linear algebra: functional analysis is linear algebra that equipped with topology. The term functional here means a mapping from vector spaces to its field.

Every Cauchy sequences in Banach space is convergent. Also, there is an orthogonality concept named Birkhoff-James. Since there is a correlation between Hilbert and Banach spaces, the question is, what is the correlation between orthogonality on Hilbert spaces and Birkhoff-James orthogonality on Banach space. As a preliminary understanding, we must know some basic concepts, such as Banach, inner product and Hilbert space and their orthogonality.

Birkhoff-James orthogonality has been studied by several authors. Arambasic [1, 2] introduced this concept in Hilbert $C^{*}$-modules. In [3], Bhattacharyya discussed its characterics. Grover in [4], told them in matrix subspace, and distance formula. Furthermore Sain [5], studied the concepts for operator norm attainment, and Paul $[6,7]$ in smoothness of linear operators.

This paper discuss orthogonality correlation of Hilbert spaces and Birkhoff-James orthogonality, also discuss minimum distance on Banach spaces.

\section{Materials and Methods}

In this literature-based project, some definitions and theorems are taken from some books and some articles. Before we discuss the main of this paper, we give some following definitions.

Definition $1[8,9,10]$ Norm is a real function $\|\cdot\|$ which maps vector space $X$ to non-negative real number such that the following requirements are satisfied:

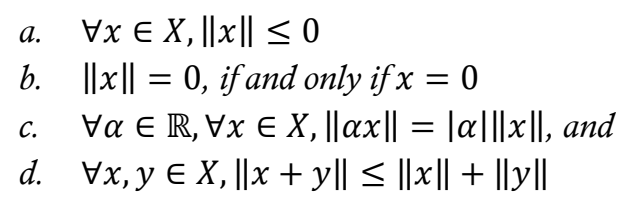


Cauchy sequence is not always convergent in a space $X$. If the Cauchy sequence in $X$ space is convergent, then the $X$ is complete. Definition 2 describes the convergence of Cauchy sequence in Banach space.

Definition 2 [8] Banach space is a complete normed space.

Inner product of vector in Euclidean space $\mathbb{R}^{n}$ is familiar for us. For our next discussion, it is explained the concept of inner product that generalized to vector space in definition 3 .

Definition 3 [8] Inner product in vector space $X$ maps from $X \times X$ to the field $K$, where each pair vector $x$ and $y$ is denoted by $\langle x, y\rangle$ and said to be an inner product of $x$ and $y$, such that each vector $x, y, z \in X$ and a scalar $\alpha$ satisfy

a. $\langle x+y, z\rangle=\langle x, z\rangle+\langle y, z\rangle$

b. $\langle\alpha x, y\rangle=\alpha\langle x, y\rangle$

c. $\langle x, y\rangle=\overline{\langle y, x\rangle}$

d. $\langle x, x\rangle \geq 0$ or $\langle x, x\rangle=0 \leftrightarrow x=0$

In definition 3, we have shown the inner product in a vector space. In the next definition we discuss a special kind of inner product space.

Definition 4 [8] Hilbert space is an inner product space whose every Cauchy sequence in ot is convergent. By other words, Hilbert space is complete space whose operator is inner product.

Some references $[10,11,12]$ called the inner product as pre-Hilbert space. So, it is equivalent to say that Hilbert space is a complete pre-Hilbert space. The correlation between Hilbert space and Banach space can be derived from Definition 2 and Definition 4 as shown in this theorem below.

Theorem 5 Let $X$ be a Hilbert space and let $\|x\|=\langle x, x\rangle^{\frac{1}{2}}$. Then $X$ is Banach spaces.

Next, from Definition 2 and Definition 4 we give some definitions about orthogonality in Hilbert space and Birkhoff-James orthogonality.

Definition 6 [8] Let $X$ be an inner product (or Hilbert) space and $x, y \in X$.If xand $y$ satisfy $\langle x, y\rangle=0$, then $x$ and $y$ are orthogonal or denoted by $x \perp y$.

Definition 6 told the orthogonality at any pre-Hilbert space. In definition 7 we show a special kind of orthogonality according to Birkhoff-James that defined on normed space (or Banach space).

Definition $7[3,7]$ Let $(X,\|\cdot\|)$ be a real Banach space, and $x, y \in X$. Then $x$ and $y$ are Birkhoff-James orthogonal (denoted by $x \perp_{B} y$ ) if satisfy

$$
\|x\| \leq\|x+\lambda y\|, \forall \lambda \in \mathbb{R}
$$

An example of the Birkhoff-James orthogonality is given below to enable us to understand the concept. Euclidean space $\left(\mathbb{R}^{2},\|\cdot\|\right)$ is also Banach space, where $\|\cdot\|$ is defined by

$$
\|x\|=\left\|\left(x_{1}, x_{2}\right)\right\|=\sqrt{x_{1}^{2}+x_{2}^{2}} ; x_{1}, x_{2} \in \mathbb{R}
$$

If we take $x=(0,1), y=(1,0) \in \mathbb{R}^{2}$, then $x \perp_{B} y$.

In definition 8, we give a definition about minimum distance in Hilbert space.

Definition 8 [10] Let $K$ be a nonempty subset in pre-Hilbert space $X$ and let $x$ be an element of $X$, and $x$ lies not in $K$. An element $y_{0} \in K$ is called the best approximation, or the nearest point to $x$ from $K$ if 


$$
\left\|x-y_{0}\right\|=d(x, K)
$$

Where $d(x, K)=\inf _{y \in K}\|x-y\|$. Thus $d(x, K)$ is the distance from $x$ to $K$, or the error in approximating $x$ by $K$.

In definition 8 , it is shown the concept of best approximation to a set $K$ from an element outside it. The uniqueness of best approximation from $x$ to $K$ is guaranteed if $K$ is convex. Ref [11] said that the convex set is Chebyshev.

\section{Results and Discussion}

In this chapter, we discuss the main question: does Birkhoff-James orthogonality stand on both Banach and Hilbert space? The correlation of definition 2, definition 4 and theorem 5 are shown below:

Theorem 9 Let $x, y \in X$ and $x \perp y$. Then $x \perp_{B} y$ where $\|x\|=\langle x, x\rangle^{\frac{1}{2}}$.

Proof: Since $x$ and $y$ are orthogonal, then $\langle x, y\rangle=0$. For all $\lambda \in \mathbb{R}$ we define $\|x+\lambda y\|=\langle x+\lambda y, x+\lambda y\rangle^{\frac{1}{2}}$. Then we obtain:

$$
\begin{aligned}
& \|x+\lambda y\|=\langle x+\lambda y, x+\lambda y\rangle^{\frac{1}{2}} \\
& \leftrightarrow\|x+\lambda y\|^{2}=\langle x+\lambda y, x+\lambda y\rangle \\
& =\langle x, x\rangle+\langle x, \lambda y\rangle+\langle\lambda y, x\rangle+\langle\lambda y, \lambda y\rangle \\
& =\langle x, x\rangle+\lambda\langle x, y\rangle+\lambda\langle y, x\rangle+\lambda^{2}\langle y, y\rangle \\
& =\langle x, x\rangle+\lambda^{2}\langle y, y\rangle
\end{aligned}
$$

Since $\|x+\lambda y\| \geq 0$ and $\|x\| \geq 0$, then

$$
\begin{aligned}
& \|x+\lambda y\|^{2} \geq\|x\|^{2} \\
& \leftrightarrow\|x+\lambda y\| \geq\|x\|
\end{aligned}
$$

Therefore, $x \perp_{B} y$.

To understand theorem 9, we give an example as shown below.

Example 1 Let $u=(x, 0), v=(y, 0)$ contained in Euclidean space $\mathbb{R}^{2}$.If $\langle u, v\rangle=u^{T} v$, then $u$ and $v$ are orthogonal according to definition 6 . The Birkhoff-James orthogonality as follows:

$$
\begin{aligned}
& \|u+\lambda v\|=\|(x, 0)+\lambda(0, y)\| \\
& =\|(x, \lambda y)\| \\
& =\sqrt{x^{2}+\lambda^{2} y^{2}}
\end{aligned}
$$

Since $\lambda^{2} y^{2} \geq 0, \forall \lambda, y \in \mathbb{R}$, then $\|u+\lambda v\| \geq \sqrt{x^{2}+0}=\|(x, 0)\|=\|u\|$. Hence $u$ and $v$ are Birkhoff-James orthogonal, or it can be written as $u \perp_{B} v$.

\section{Minimum Distance in Banach Space}

In this section, we discuss about the theorem of minimum distance in Banach space. Ref [10] proposed theorem 10, and we generalize it to Banach space.

Theorem 10 Suppose $x$ be a vector in a Banach space $X$ and $K$ is a closed convex subset in $X$, then

$$
\left\|x-k_{0}\right\| \leq\|x-k\|
$$

$k_{0} \in K$ is a unique vector for all $k \in K$. Furthermore, a necessary and sufficient condition that $k_{0}$ be the unique minimizing vector is $\left\langle x-k_{0}, k-k_{0}\right\rangle \leq 0, \forall k \in K$.

Proof: To prove the existence, let $\left\{k_{i}\right\}$ be a sequence in $K$ such that

$$
\left\|x-k_{i}\right\| \rightarrow \delta=\inf _{k \in K}\|x-k\| .
$$


By the parallelogram law,

$$
\left\|k_{i}-k_{j}\right\|^{2}=2\left\|k_{i}-x\right\|^{2}+2\left\|k_{j}-x\right\|^{2}-4\left\|x-\frac{k_{i}+k_{j}}{2}\right\|^{2}
$$

Since $K$ is convex and $k_{i}, k_{j} \in K$, then $\frac{k_{i}+k_{j}}{2} \in K$. Hence

And therefore

$$
\left\|x-\frac{k_{i}+k_{j}}{2}\right\| \geq \delta
$$

$$
\begin{aligned}
& \left\|k_{i}-k_{j}\right\|^{2}=2\left\|k_{i}-x\right\|^{2}+2\left\|k_{j}-x\right\|^{2}-4\left\|x-\frac{k_{i}+k_{j}}{2}\right\|^{2} \\
& \leq 2\left\|k_{i}-x\right\|^{2}+2\left\|k_{j}-x\right\|^{2}-4 \delta^{2} \rightarrow 0
\end{aligned}
$$

Then $\left\{k_{i}\right\}$ is Cauchy sequence, and it is convergent to $k_{0} \in K$. In other words, by continuity we obtain that $\left\|x-k_{0}\right\|=\delta$.

Next, to show the uniqueness, suppose there is $k_{i} \in K$ with $\left\|x-k_{1}\right\|=\delta$. The sequence

$$
k_{n}=\left\{\begin{array}{lc}
k_{0}, & n \text { odd } \\
k_{1}, & n \text { even }
\end{array}\right.
$$

Also convergent with $\left\|x-k_{n}\right\| \rightarrow \delta$. Hence if $k_{0}=k_{1}$ attained, then $\left\{k_{i}\right\}$ is Cauchy and convergent.

For the last, we prove that if $k_{0}$ is the unique minimizing vector, then

$$
\left\langle x-k_{0}, k-k_{0}\right\rangle \leq 0
$$

For all $k \in K$. Suppose the contrary: there is a vector $k_{1} \in K$ where $\left\langle x-k_{0}, k_{1}-k_{0}\right\rangle=\varepsilon>0$. Consider the vectors $k_{\alpha}=(1-\alpha) k_{0}+\alpha k_{1}$, with $0 \leq \alpha \leq 1$. Since $K$ is convex, each $k_{\alpha} \in K$. Also

$$
\begin{aligned}
& \left\|x-k_{\alpha}\right\|^{2}=\left\|(1-\alpha)\left(x-k_{0}\right)+\alpha\left(x-k_{1}\right)\right\|^{2} \\
& =(1-\alpha)^{2}\left\|x-k_{0}\right\|^{2}+2 \alpha(1-\alpha)\left\langle x-k_{0}, x-k_{1}\right\rangle+\alpha^{2}\left\|x-k_{1}\right\|^{2}
\end{aligned}
$$

At $\alpha=0$, the function $\left\|x-k_{\alpha}\right\|^{2}$ is differentiable that equal to

$$
\begin{gathered}
\left.\frac{d y}{d x}\left\|x-k_{\alpha}\right\|^{2}\right|_{\alpha=0}=-2\left\|x-k_{0}\right\|^{2}+2\left\langle x-k_{0}, x-k_{1}\right\rangle \\
=-2\left\langle x-k_{0}, k_{1}-k_{0}\right\rangle=-2 \varepsilon<0 .
\end{gathered}
$$

Thus for any small positive $\alpha$, then $\left\|x-k_{\alpha}\right\|<\left\|x-k_{0}\right\|$ that contradicts to the minimizing property of $k_{0}$. Hence, there no exist $k_{1}$. Conversely, suppose that $k_{0} \in K$ is such that $\left\langle x-k_{0}, k-k_{0}\right\rangle \leq 0$ for all $k \in$ $K$. Then for any $k \in K, k \neq k_{0}$, we have

$$
\begin{aligned}
& \|x-k\|^{2}=\left\|x-k_{0}+k_{0}-k\right\|^{2} \\
& =\left\|x-k_{0}\right\|^{2}+2\left\langle x-k_{0}, k_{0}-k\right\rangle+\left\|k_{0}-k\right\|^{2}>\left\|x-k_{0}\right\|^{2}
\end{aligned}
$$

From theorem 10, we have shown that condition $\left\langle x-k_{0}, k-k_{0}\right\rangle=0$ satisfies the Birkhoff-James orthogonality.

Theorem 11 Condition $\left\langle x-k_{0}, k-k_{0}\right\rangle=0$ in theorem 10 satisfies the Birkhoff-James orthogonality with $\|x\|=$ $\langle x, x\rangle^{\frac{1}{2}}$.

Proof: Let $\left(x-k_{0}\right),\left(k-k_{0}\right) \in X$. For all $\lambda \in \mathbb{R}$, we define

$$
\left\|\left(x-k_{0}\right)+\lambda\left(k-k_{0}\right)\right\|=\left\langle\left(x-k_{0}\right)+\lambda\left(k-k_{0}\right),\left(x-k_{0}\right)+\lambda\left(k-k_{0}\right)\right)^{\frac{1}{2}} .
$$

Then we obtain

$$
\begin{aligned}
& \left\|\left(x-k_{0}\right)+\lambda\left(k-k_{0}\right)\right\|=\left\langle\left(x-k_{0}\right)+\lambda\left(k-k_{0}\right),\left(x-k_{0}\right)+\lambda\left(k-k_{0}\right)\right\rangle^{\frac{1}{2}} \\
& \leftrightarrow\left\|\left(x-k_{0}\right)+\lambda\left(k-k_{0}\right)\right\|^{2}=\left\langle\left(x-k_{0}\right)+\lambda\left(k-k_{0}\right),\left(x-k_{0}\right)+\lambda\left(k-k_{0}\right)\right\rangle \\
& =\left\langle\left(x-k_{0}\right),\left(x-k_{0}\right)\right\rangle+\left\langle\left(x-k_{0}\right), \lambda\left(k-k_{0}\right)\right\rangle+\left\langle\lambda\left(k-k_{0}\right),\left(x-k_{0}\right)\right\rangle+\left\langle\lambda\left(k-k_{0}\right), \lambda\left(k-k_{0}\right)\right\rangle
\end{aligned}
$$




$$
\begin{aligned}
& =\left\langle\left(x-k_{0}\right),\left(x-k_{0}\right)\right\rangle+\lambda\left\langle\left(x-k_{0}\right),\left(k-k_{0}\right)\right\rangle+\lambda\left\langle\left(k-k_{0}\right),\left(x-k_{0}\right)\right\rangle+\lambda^{2}\left\langle\left(k-k_{0}\right),\left(k-k_{0}\right)\right\rangle \\
& =\left\langle\left(x-k_{0}\right),\left(x-k_{0}\right)\right\rangle+\lambda^{2}\left\langle\left(k-k_{0}\right),\left(k-k_{0}\right)\right\rangle \\
& \geq\left\langle\left(x-k_{0}\right),\left(x-k_{0}\right)\right\rangle=\left\|\left(x-k_{0}\right)\right\|^{2}
\end{aligned}
$$

Since $\left\|\left(x-k_{0}\right)+\lambda\left(k-k_{0}\right)\right\| \geq 0$ and $\left\|\left(x-k_{0}\right)\right\|^{2} \geq 0$, then

$$
\begin{aligned}
& \left\|\left(x-k_{0}\right)+\lambda\left(k-k_{0}\right)\right\|^{2} \geq\left\|\left(x-k_{0}\right)\right\|^{2} \\
& \leftrightarrow\left\|\left(x-k_{0}\right)+\lambda\left(k-k_{0}\right)\right\| \geq\left\|\left(x-k_{0}\right)\right\|
\end{aligned}
$$

Therefore, condition $\left\langle\left(x-k_{0}\right),\left(k-k_{0}\right)\right\rangle=0$ satisfies the Birkhoff-James orthogonality. We need a further discussion about the Birkhoff-James orthogonality for $\left\langle\left(x-k_{0}\right),\left(k-k_{0}\right)\right\rangle<0$.

\section{Conclusion}

After some discussions, we have the conclusion below

1. Correlation of Hilbert space and Birkhoff-James orthogonality have been found in theorem 10 .

2. The minimum distance condition $\left\langle\left(x-k_{0}\right),\left(k-k_{0}\right)\right\rangle=0$ satisfies in the Birkhoff-James orthogonality.

3. For condition $\left\langle\left(x-k_{0}\right),\left(k-k_{0}\right)\right\rangle<0$, set this problem as an open problem for further discussion.

\section{References}

[1] L. Arambasic and R. Rajic, "The Birkhoff-James Orthogonality in Hilbert C*-modules," Linear Algebra, vol. 437, pp. 1913-1929, 2012.

[2] L. Arambasic and R. Rajic, "A Strong Version of the Birkhoff-James Orthogonality in Hilbert C*modules," Annals of Functinal Analysis, vol. 1, pp. 109-120, 2014.

[3] T. Bhattacharyya and P. Grover, "Characterization of Birkhoff-James Orthogonality," Journal of Mathematical Analysis and Applications, vol. 407, pp. 350-358, 2013.

[4] P. Grover, "Orthogonality to Matrix Subspaces, and a Distance Formula," Linear Algebra, vol. 445, pp. 280-288, 2014.

[5] D. Sain, K. Paul and S. Hait, "Operator Norm Attainment and Birkhoff-James Orthogonality," Linear Algebra, vol. 476, pp. 85-97, 2015.

[6] K. Paul, D. Sain and P. Ghosh, "Birkhoff-James Orthogonality and Smoothness of Bounded Linear Operators," Linear Algebra, vol. 506, pp. 551-563, 2016.

[7] P. Ghosh, D. Sain and K. Paul, "On Symmetry of Birkhoff-James Orthogonality of Linear Operators," Advance in Operator Theory, vol. 4, pp. 428-434, 2017.

[8] E. Kreyszig, Introductory Functional Analysis with Applications, New York: John Wiley \& Sons, Inc, 1978.

[9] A. Dax, "The Distance between Two Convex Sets," Linear Algebra and its Applications, pp. 184-213, 2006.

[10] A. Iske, Approximation Theory and Algorithms for Data Analysis, Switzerland: Springer Nature Switzerland, 2010.

[11] D. G. Luenberger, Optimization by Vector Space Methods, New York: John Wiley \& Sons, Inc, 1969.

[12] F. Deutsch, Best Approximation in Inner product Spaces, New York: Springer Verlag, Inc, 2001.

[13] J. Weidmann, Linear Operator in Hilbert Spaces, New York: Springer-Verlag New York, 1980. 